\title{
EGU2020-12986
}

https://doi.org/10.5194/egusphere-egu2020-12986

EGU General Assembly 2020

(c) Author(s) 2021. This work is distributed under

the Creative Commons Attribution 4.0 License.

\section{First Field-Test results of iGNSS-R instrument of the PRETTY payload}

Andreas Dielacher ${ }^{1}$, Heinz Fragner ${ }^{1}$, Michael Moritsch ${ }^{1}$, Jens Wickert ${ }^{2,3}$, Otto Koudelka ${ }^{4}$, Per Hoeg ${ }^{5}$, Estel Cardellach ${ }^{6}$, Manuel Martin-Neira ${ }^{7}$, Maximilian Semmling ${ }^{2,8}$, Roger Walker ${ }^{7}$, Andreas Hörmer ${ }^{4}$, and Manuela Wenger ${ }^{4}$

${ }^{1}$ RUAG Space GmbH, Vienna, Austria (andreas.dielacher@ruag.com)

${ }^{2}$ German Research Centre for Geosciences (GFZ), Potsdam, Germany

${ }^{3}$ Technische Universität Berlin, Germany

${ }^{4}$ Graz University of Technology, IKS, Graz, Austria

${ }^{5}$ University of Oslo (UiO), Department of Physics, Oslo, Norway

${ }^{6}$ Institute of Space Sciences (ICE-CSIC) and Institut d'Estudis Espacials de Catalunya (IEEC), Barcelona, Spain

${ }^{7}$ European Space Agency (ESA), Noordwijk, The Netherlands

${ }^{8}$ Institute for Solar-terrestrial Physics (DLR-SO), Neustrelitz, Germany

The PRETTY mission is a $3 \mathrm{U}$ CubeSat mission, hosting two different payloads, a radiation dosimeter and an interferometric GNSS reflectometer. The intended launch is planned in 2022.

The reflectometer payload has been built, using flight representative hardware and mounted inside a portable setup. Two campaigns have been carried out, a first one to verify the setup in real world condition and the second one to record reflectometry data over the Danube river. The reflections over the river are analyzed and compared to a reference data set obtained from basemap.at (which is released under Open Government Data Österreich Lizenz CC-BY 4.0).

The hardware is capable of generating complex and power waveforms at the same time, and the reflection events are visible in both. Since PRETTY is aiming for phase altimetry, only coherent measurements are conducted with an integration time of $20 \mathrm{~ms}$.

The re-tracking algorithm for the specular point and height estimation are based on [1]. Due to the low elevation angle and receiver height, the effects from the ionosphere is not considered, however effects from the atmosphere have to be included in the data re-tracking process. The reflection peaks, and the signal to noise ratio of the peaks, are large enough detect the peak and to calculate the height of the reflection point. The height retrieval is shown in the paper.

The results are promising w.r.t. the performance of the overall structure of the PRETTY GNSS-R payload in order to deliver altimetry results on a low-cost CubeSat platform.

[1] W. Li, E. Cardellach, F. Fabra, S. Ribó and A. Rius, "Assessment of Spaceborne GNSS-R Ocean Altimetry Performance Using CYGNSS Mission Raw Data," in IEEE Transactions on Geoscience and Remote Sensing, vol. 58, no. 1, pp. 238-250, Jan. 2020. doi: 10.1109/TGRS.2019.2936108 
